\title{
Search for the gauge boson of a secluded sector with the PADME experiment at LNF
}

\author{
P. Gianotti on behalf of the PADME Collaboration ${ }^{* \dagger}$ \\ Laboratori Nazionali di Frascati dell'INFN \\ Via E. Fermi 40, I-00044 Frascati (Italy) \\ E-mail: paola.gianottielnf.infn.it
}

\begin{abstract}
Massive photon-like particles are predicted in many extensions of the Standard Model with a hidden sector where dark matter is secluded. Most of the present experimental constraints on this "dark photon" $\left(A^{\prime}\right)$ rely on the hypothesis of dominant decay to lepton pairs. The PADME experiment at Laboratori Nazionali di Frascati of INFN, by annihilating the positrons of the DAФNE Beam-Test Facility on a carbon target, will search for the $\mathrm{e}^{+} \mathrm{e}^{-} \rightarrow \gamma \mathrm{A}^{\prime}$ process, assuming a decay of the $A^{\prime}$ into invisible particles of the hidden sector. A fine-grained, high-resolution calorimeter will measure the momentum of the photon in events with no other signal in the apparatus, thus allowing to identify the $A^{\prime}$ as a missing mass in the final state. In about one year of data taking, a sensitivity on the interaction strength $(\varepsilon)$ down to 0.001 is achievable in the mass region $\mathrm{M}\left(\mathrm{A}^{\prime}\right)$ $<23.7 \mathrm{MeV} / c^{2}$. The status of PADME and its physics potential are here reviewed.
\end{abstract}

The European Physical Society Conference on High Energy Physics

5-12 July, 2017

Venice

* Speaker.

${ }^{\dagger}$ G. Chiodini, P. Creti, F. Oliva (INFN Lecce), A.P. Caricato, M. Martino, G. Maruccio, A. Monteduro, V. Scherini, S.Spagnolo (INFN Lecce e Dip. di Matematica e Fisica, Università del Salento), P. Albicocco, R. Bedogni, B. Buonomo, F. Bossi, R. De Sangro, G. Finocchiaro, L.G. Foggetta, A. Ghigo, P. Gianotti, M. Palutan, G. Piperno, I. Sarra, B. Sciascia, T. Spadaro, E. Spiriti (INFN Laboratori Nazionali di Frascati), L. Tsankov, (University of Sofia "St. Kl. Ohridski"), G. Georgiev, V. Kozhuharov (University of Sofia "St. Kl. Ohridski" and INFN Laboratori Nazionali di Frascati), F. Ameli, F. Ferrarotto, E. Leonardi, P. Valente (INFN Roma1), S. Fiore (INFN Roma1 e ENEA), G.C.Organtini, M.Raggi (INFN Roma1 e Dip. di Fisica, "Sapienza" Università di Roma), C. Taruggi (INFN Laboratori Nazionali di Frascati e Dip. di Fisica, Università di Roma "Tor Vergata"). 


\section{Introduction}

Direct observations of dark matter signals are necessary to shed light on the cosmological and astrophysical evidences, in order to understand the nature of such unknown matter that at present has manifested only via gravitational interactions.

One of the present theoretical hypothesis foresees that the dark particles are not directly connected with the Standard Model (SM) gauge fields, but only through mediators or "portals", linking our world with the new "secluded" sector [1]. A simple class of models that try to explain dark matter, just adds an additional $U(1)$ symmetry, with its corresponding vector boson $A^{\prime}$. All SM particles are neutral under this symmetry, and the new field couples to the charged particles of the SM with an effective charge $\varepsilon q$, where $q$ is the electromagnetic one, induced by the kinetic mixing of the dark photon with the SM photon.

At Laboratori Nazionali di Frascati (LNF) of INFN is in preparation an experiment, aiming at exploring this field, that in spring 2018 will start the data taking. By annihilating $\sim 10^{13}$ positrons of $550 \mathrm{MeV} / c$ on target, it will reach a sensitivity $\varepsilon \sim 10^{-3}$ on the coupling of dark photons with masses up to $23.7 \mathrm{MeV} / c^{2}$.

\section{The PADME experiment}

To test the hypothesis of the existence of a dark photon in the low energy range, PADME (Positron Annihilation into Dark Matter Experiment) will search for invisible decays of the $A^{\prime}$ at LNF by using the positrons of the DAФNE LINAC. The experiment is designed to measure the missing mass of the reaction

$$
e^{+} e^{-} \rightarrow \gamma A^{\prime}
$$

Knowing the momentum of the positron, with the target electrons assumed at rest $\left(P_{e^{-}}=0\right)$, and measuring the annihilation point and the 4-momentum of the recoil photon by means of a finely segmented electromagnetic calorimeter, the mass of the missing state $A^{\prime}$ can be evaluated:

$$
M_{\text {miss }}^{2}=\left(P_{\text {beam }}+P_{e^{-}}-P_{\gamma}\right)^{2}
$$

The detector setup (shown in fig. 1) consists of the following elements:

- a $100 \mu \mathrm{m}$ tick diamond active target, to measure the average position and the intensity of the positrons. It consists of a polycrystalline carbon film $\left(20 \times 20 \mathrm{~mm}^{2}\right)$ on which $16 \times 1 \mathrm{~mm}^{2}$ graphitized strips have been realized with a laser technique on both sides. The strips have different orientation (X-Y) to allow a 2-dimensional readout. A prototype of the active target was fully characterised with the electron beam of the LNF Beam Test Facility, showing a good position resolution $(\sim 0.2 \mathrm{~mm})$ for both coordinates, and timing performance $\sim 2 \mathrm{~ns}$ meeting the experiment needs [2]. To further improve beam monitor capability, a movable silicon pixel detector, based on MIMOSA 28 Ultimate chips, is under construction [3]. These chips consist of a matrix of $928 \times 960$ pixels of size $20.7 \mu \mathrm{m}$, and have a thickness of $50 \mu \mathrm{m}$;

- a magnetic field to precisely measure the momentum of the not interacting positrons before deflecting them on a beam dump. This is realized with a dipole producing a field of $0.55 \mathrm{~T}$ having a gap of $23 \mathrm{~cm}$; 


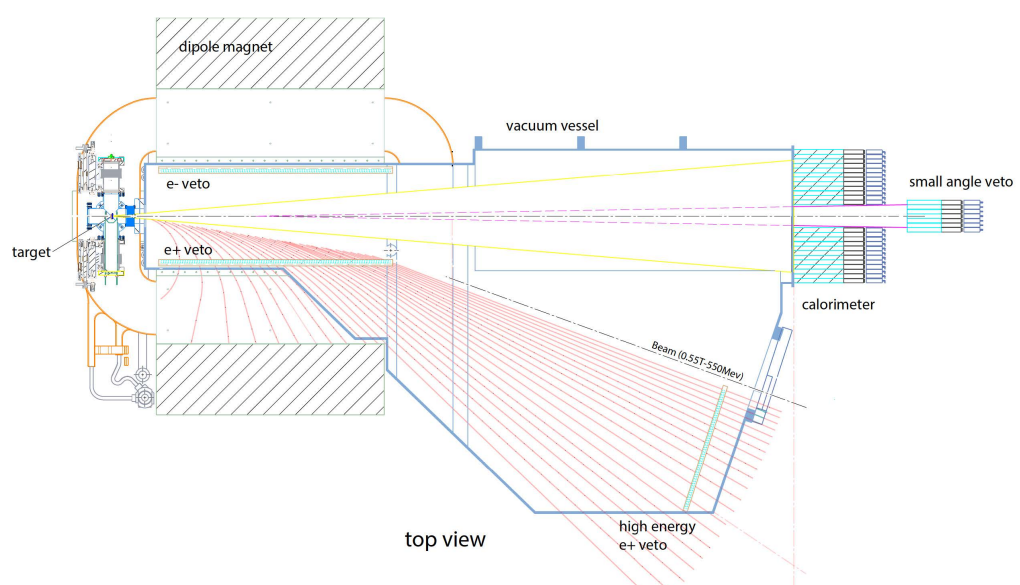

Figure 1: Layout of the PADME experiment (see text for more details).

- a set of plastic scintillators, used to veto the positrons/electrons losing their energy due to Bremsstrahlung, installed on the side and the forward walls of a vacuum chamber placed in the gap of the dipole magnet. The vacuum chamber is necessary in order to minimize unwanted interactions of primary and secondary particles;

- an electromagnetic calorimeter, to measure final state photons, placed $\sim 3 \mathrm{~m}$ downstream the production target. It consists in an array of 616 BGO crystals arranged in a cylindrical shape, readout by photomultipliers. The small size of the crystals $\left(21 \times 21 \times 230 \mathrm{~mm}^{3}\right)$, which are reshaped blocks of the endcap e.m. calorimeter of the L3 experiment, allow to reach $\sim 5 \mathrm{~mm}$ on the position resolution for electromagnetic clusters determining a precise measurement of the direction of the impinging photon. The energy resolution, measured using the electron beam of the LNF, is $\frac{\sigma(E)}{E}=\left(\frac{2}{\sqrt{E}(\mathrm{GeV})} \oplus 1\right) \%$ [4]. The calorimeter will have a central hole $\left(100 \times 100 \mathrm{~mm}^{2}\right)$ through which the majority of the Bremsstrahlung photons will pass;

- a small angle faster calorimeter, to veto background photons emitted in the forward region. Based on Cherenkov crystals, it will be placed in the shadow of the central hole of the main calorimeter. It will be an array of 25 elements $\left(30 \times 30 \times 150 \mathrm{~mm}^{3}\right)$ of $\mathrm{PbF}_{2}$ read out by fast $(<0.6$ ns rise time) photomultipliers.

\section{Signal and background}

The sensitivity of the PADME experiment has been evaluated using a detailed Monte Carlo simulation based on GEANT4. It reproduces the present characteristics of the LNF positron beam: $40 \mathrm{~ns}$ bunch length; 5000 particles per bunch; momentum $550 \mathrm{MeV} / \mathrm{c}$ with a spread of $1 \%$; beam angular divergence of $\sim 1 \mathrm{mrad}$; Gaussian beam spot with $\sigma_{x, y}=1 \mathrm{~mm}$. With this setup, it will be possible to integrate a luminosity of $10^{13}$ positrons on target in roughly two years of data taking, assuming an efficiency of 50\%. Nevertheless, some upgrades performed on the DAФNE LINAC allow to extend bunch duration. This will make possible to reduce the time to collect this statistics. 
The simulation considers also background events. By using GEANT4 low-energy electromagnetic libraries, two photon annihilation, ionization processes, Bhabha and Møller scattering, and the production of $\delta$-rays are included. To simulate the $A^{\prime}$ production and its decays into $\mathrm{e}^{+} \mathrm{e}^{-}$or invisible particles, and to account for the three photons annihilation background, custom generators have been developed.

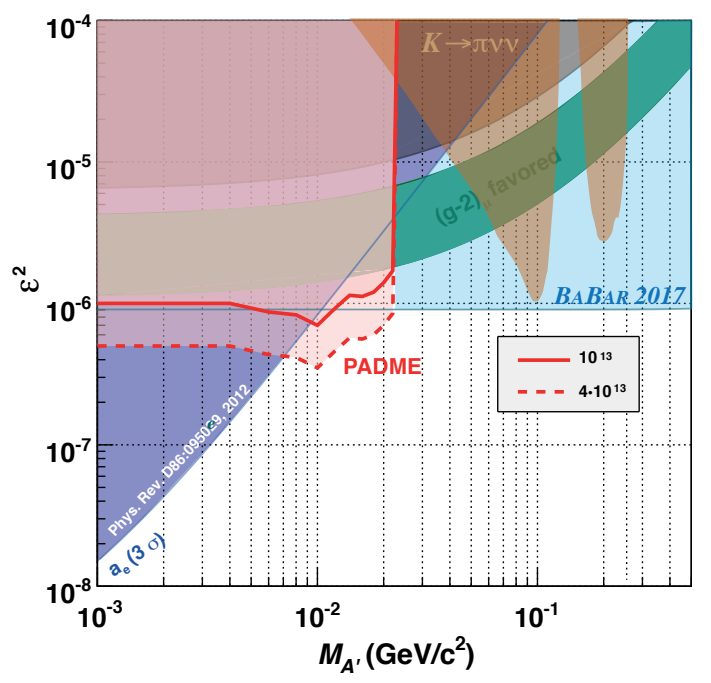

Figure 2: The PADME experiment expected sensitivity.

The results of the simulations are shown in fig. 2. The expected sensitivity of PADME to the invisible decays of the dark photon is shown together with the results of other experiments.

The PADME physics potential extends beyond dark photon search. Presently, the sensitivity estimate is available only for the invisible dark photon decays, but the detector will be sensitive to any new light particle produced in the annihilation process: i.e. Axion Like Particles, Dark Higgs, etc.

\section{Conclusions}

The search for dark photon signals are well motivated within several new physics scenarios. The PADME experiment at LNF will be able to contribute to this search in just one-two years of data taking with a sensitivity on the $A^{\prime}$ mass up to $23.7 \mathrm{MeV} / c^{2}$ in a complete model independent way.

This work is partly supported by the project PGR-226 of the Italian Ministry of Foreign Affairs and International Cooperation (MAECI), CUP I86D16000060005.

\section{References}

[1] B. Holdom, Phys. Lett. B166, 196 (1986).

[2] G. Chiodini, JINST 12, C02036 (2017).

[3] I. Valin et al., JINST 7, C01102 (2012).

[4] M. Raggi et al., Nucl. Inst. Meth A 862, 31 (2017). 